\title{
STEINER MINIMAL TREES ON ZIG-ZAG LINES
}

\author{
BY
}

D. Z. DU, F. K. HWANG AND J. F. WENG

\begin{abstract}
A Steiner minimal tree for a given set $P$ of points in the Euclidean plane is a shortest network interconnecting $P$ whose vertex set may include some additional points. The construction of Steiner minimal trees has been proved to be an $N P$-complete problem for general $P$. However, the $N P$-completeness does not exclude the possibility that Steiner trees for sets of points with special structures can be efficiently determined. In this paper we determine the Steiner minimal trees for zig-zag lines with certain regularity properties. We also give an explicit formula for the length of such a tree.
\end{abstract}

1. Introduction. Let $P$ denote a given set of points in the Euclidean plane. A minimal spanning tree for $P$ is the shortest network (clearly, it has to be a tree) whose vertex set is $P$. A Steiner minimal tree (SMT) for $P$ is the shortest network (again, it must be a tree) interconnecting $P$ whose vertex set may include some additional points, called Steiner points, other than points in $P$ (called regular points).

While there exist efficient algorithms for constructing minimal spanning trees, the construction of SMT's has been proved [3] to be an NP-complete problem. However, the NP-completeness does not exclude the possibility that the SMT's for sets of points with special structures can be efficiently determined. One such class was recently studied by Chung and Graham [2]. We quote:

Perhaps the simplest infinite family of sets whose SMT's one might study are the ladders, so named by Boyce, who first focussed attention on them in [1]. A ladder $L_{n}$ consists of $2 n$ points arranged in a rectangular 2 by $n$ array with adjacent pairs of points forming a square.

In their paper Chung and Graham determined the SMT's for $L_{n}$. However, even for sets of points with this extremely simple structure, it takes almost 30 pages [2] just to give a sketch of the proof. In this paper we study another class of sets of points for which SMT's can be determined with surprising ease.

A zig-zag line is a connected sequence of line segments $\left[p_{1}, p_{2}\right]$, $\left[p_{2}, p_{3}\right], \ldots,\left[p_{n-1}, p_{n}\right]$ which turn in alternate directions (see Figure 1). A zig-zag line is called regular if $\Varangle p_{i} p_{i+1} p_{i+2}$ is constant for all $i=1,2, \ldots, n-2$. We will denote such a zig-zag line by $Z\left(P_{n}, \alpha\right)$ where $P_{n}=\left\{p_{1}, p_{2}, \ldots, p_{n}\right\}$ and $\alpha<180^{\circ}$ is the constant angle. A zig-zag line is called convex if the points $p_{i}$ are the vertices of a convex polygon. Note that for a convex zig-zag line, the line $\left[p_{1}, p_{n}\right]$ cuts through line $\left[p_{i}, p_{i+1}\right]$ for all $i$. A zig-zag line is called normal if $\left|p_{i}, p_{i+1}\right| \leqslant\left|p_{i}, p_{i+2}\right|$ for $1 \leqslant i \leqslant n-3$ and $\left|p_{i+1}, p_{i+2}\right| \leqslant\left|p_{i}, p_{i+2}\right|$ for $2 \leqslant i \leqslant n-2$, where $|p, q|$ denotes

Received by the editors January 22, 1982 and, in revised form, April 28, 1882.

1980 Mathematics Subject Classification. Primary 51M15; Secondary 90B99, 05C40. 
the length of $[p, q]$. Note that if $Z\left(P_{n}, \alpha\right)$ is a minimal spanning tree for $P_{n}$, then $Z\left(P_{n}, \alpha\right)$ is normal (the converse is not true). Also note that being normal implies $\alpha \geqslant 60^{\circ}$, and if $\alpha=60^{\circ}$, then $\left|p_{2}, p_{3}\right|=\left|p_{3}, p_{4}\right|=\cdots=\left|p_{n-2}, p_{n-1}\right|$.

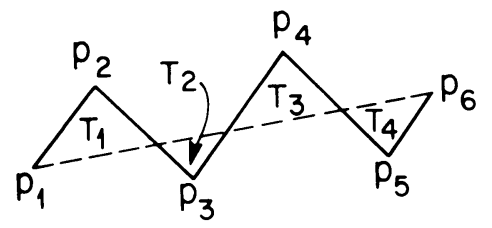

Figure 1. A convex zig-zag line

We now prove some properties of convex or normal $Z\left(P_{n}, \alpha\right)$.

Lemma 1. Let $q$ be a point on $\left[p_{i}, p_{i+1}\right]$ of $Z\left(P_{n}, \alpha\right)$. Define $P^{\prime}=\left\{p_{1}, \ldots, p_{i}, q\right\}$ and $P^{\prime \prime}=\left\{q, p_{i+1}, \ldots, p_{n}\right\}$. If $Z\left(P_{n}, \alpha\right)$ is convex, so are $Z\left(P^{\prime}, \alpha\right)$ and $Z\left(P^{\prime \prime}, \alpha\right)$. If $Z\left(P_{n}, \alpha\right)$ is normal, so are $Z\left(P^{\prime}, \alpha\right)$ and $Z\left(P^{\prime \prime}, \alpha\right)$.

Proof. Immediate from the definitions.

It is interesting to note that if $Z\left(P_{n}, \alpha\right)$ is a minimal spanning tree for $P_{n}$, then $Z\left(P^{\prime}, \alpha\right)$ and $Z\left(P^{\prime \prime},\langle)\right.$ need not be minimal spanning trees for $P^{\prime}$ and $P^{\prime \prime}$, respectively.

Lemma 2. Suppose that $Z\left(P_{n}, \alpha\right)$ is normal. Then for $1 \leqslant i \leqslant n-3$

$$
\left|p_{i}, p_{i+1}\right| \leqslant\left|p_{i}, p_{j}\right| \text { for all } i+2 \leqslant j,
$$

where the equality is possible only when $j=i+2$.

Proof. $\left|p_{i}, p_{i+1}\right| \leqslant\left|p_{i}, p_{i+2}\right|$ by the definition of "normal". For $i+3 \leqslant j$, let $\left[p_{i}, p_{j}\right]$ meet $\left[p_{i+2}, p_{i+3}\right]$ at $q$ (see Figure 2).

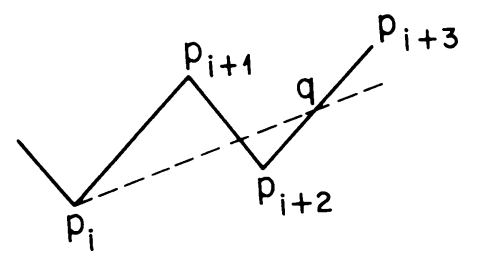

Figure 2. A normal zig-zag line

Then

$$
\Varangle p_{i+1} q p_{i+2} \leqslant 180^{\circ}-\alpha \leqslant 120^{\circ} .
$$

Therefore either $\Varangle p_{i+1} q p_{i}<60^{\circ} \leqslant \alpha<\Varangle q p_{i+1} p_{i}$, hence $\left|p_{i}, p_{i+1}\right|<\left|p_{i}, q\right| \leqslant$ $\left|p_{i}, p_{j}\right|$, or $\Varangle p_{i+2} q p_{i} \leqslant 60^{\circ} \leqslant \alpha<\Varangle q p_{i+1} p_{i}$, hence

$$
\left|p_{i}, p_{i+1}\right| \leqslant\left|p_{i}, p_{i+2}\right|<\left|p_{i}, q\right| \leqslant\left|p_{i}, p_{j}\right| \text {. }
$$

Let $T_{1}, T_{2}, \ldots, T_{n-2}$ be the triangles formed by a convex $Z\left(P_{n}, \alpha\right)$ and $\left[p_{1}, p_{n}\right]$ (see Figure 1). Let $S_{i}$ be the SMT for the three vertices of $T_{i}$ and let $S=\cup_{i=1}^{n-2} S_{i}$. In this paper we prove that if $Z\left(P_{n}, \alpha\right)$ is convex and normal, then $S$ is its SMT. We also give an explicit formula for the length of $S$. 
2. Some preliminary results. Lemmas $3-7$ are all well known in the literature $[4,5]$. Let $T$ denote an SMT.

Lemma 3. No two edges in $T$ incident to a vertex can form an angle less than $120^{\circ}$.

LEMMA 4. Each Steiner point in Thas three edges meeting at $120^{\circ}$.

Lemma 5. T lies in the convex hull of $P_{n}$.

LEMma 6. Let $A, B$ and $C$ be the three vertices of a triangle which has no angle exceeding $120^{\circ}$. Then the length of an SMT for these three points is

$$
\left[|A, B|^{2}+|B, C|^{2}-2|A, B||B, C| \cos \left(60^{\circ}+\Varangle A B C\right)\right]^{1 / 2} .
$$

Lemma 7. Suppose that $T$ does not contain the edge $\left[p_{i}, p_{j}\right]$. Then $\left|p_{i}, p_{j}\right| \geqslant$ the length of any edge on the path joining $p_{i}$ and $p_{j}$ in the tree.

We also quote a result from Pollak [6].

Lemma 8. Let four points $A, B, C, D$ be given such that both possible full Steiner trees actually exist. Then the shorter of the two is in the same direction as the acute angle between the diagonals of the quadrilateral $A B C D$. The two full Steiner trees (given they both exist) will be equally long if and only if the diagonals are perpendicular.

(A full Steiner tree with $n$ given points is an SMT with $n-2$ Steiner points.)

Let $x_{i}$ denote $\left|p_{2 i-1}, p_{2 i}\right|$ and $y_{i}$ denote $\left|p_{2 i}, p_{2 i+1}\right|$. We will suppress the subscript $i$ when taking the sum over all $i$. Define $L_{S}\left(P_{n}, \alpha\right)$ to be the length of $S$.

THEOREM 1. If $Z\left(P_{n}, \alpha\right)$ is convex and $60^{\circ} \leqslant \alpha \leqslant 120^{\circ}$, then

$$
L_{S}\left(P_{n}, \alpha\right)=\left[\left(\sum x\right)^{2}+\left(\sum y\right)^{2}-2\left(\sum x\right)\left(\sum y\right) \cos \left(60^{\circ}+\alpha\right)\right]^{1 / 2}
$$

Proof. Theorem 1 is reduced to Lemma 6 for $n=3$. We prove the general case by induction on $n$. For $n>3$, let $\left[p_{1}, p_{n}\right]$ meet $\left[p_{2}, p_{3}\right]$ at $q$. It is straightforward to verify $\left|p_{2}, q\right|=x_{1}(\Sigma y)(\Sigma x)^{-1}$. Therefore

$$
\begin{aligned}
& L_{S}\left(P_{n}, \alpha\right)=L_{S}\left(\left\{p_{1}, p_{2}, q\right\}, \alpha\right)+L_{S}\left(P_{n}-\left\{p_{1}, p_{2}\right\} \cup\{q\}, \alpha\right) \\
& =\left[x_{1}^{2}+x_{1}^{2}\left(\sum y\right)^{2}\left(\sum x\right)^{-2}-2 x_{1}^{2}\left(\sum y\right)\left(\sum x\right)^{-1} \cos \left(60^{\circ}+\alpha\right)\right]^{1 / 2} \\
& \quad+\left[\left(\sum x-x_{1}\right)^{2}+\left\{\sum y-x_{1}\left(\sum y\right)\left(\sum x\right)^{-1}\right\}^{2}-2\left(\sum x-x_{1}\right)\right. \\
& \left.\cdot\left\{\sum y-x_{1}\left(\sum y\right)\left(\sum x\right)^{-1}\right\} \cos \left(60^{\circ}+\alpha\right)\right]^{1 / 2} \\
& =\left(x_{1}+\sum x-x_{1}\right)\left[1+\left(\sum y\right)^{2}\left(\sum x\right)^{-2}-2\left(\sum y\right)\left(\sum x\right)^{-1} \cos \left(60^{\circ}+\alpha\right)\right]^{1 / 2} \\
& =\left[\left(\sum x\right)^{2}+\left(\sum y\right)^{2}-2\left(\sum x\right)\left(\sum y\right) \cos \left(60^{\circ}+\alpha\right)\right]^{1 / 2} \cdot \square
\end{aligned}
$$


Theorem 2. Let $Z\left(P_{n}, \alpha\right)$ be convex, $60^{\circ} \leqslant \alpha<120^{\circ}$. Suppose that $q$ is a point on $\left[p_{i}, p_{i+1}\right]\left(q\right.$ can be $\left.p_{i}\right)$. Then for $n-2 \geqslant i \geqslant 2$,

$$
f(q)=L_{S}\left(\left\{p_{1}, p_{2}, \ldots, p_{i}, q\right\}, \alpha\right)+L_{S}\left(\left\{q, p_{i+1}, p_{i+2}, \ldots, p_{n}\right\}, \alpha\right)
$$

is minimized when $q$ is on $\left[p_{1}, p_{n}\right]$. Hence the minimum is equal to $L_{S}\left(P_{n}, \alpha\right)$.

Proof. We first prove Theorem 2 for $i=2$. Let $z=\left|p_{2} q\right|$.

$$
\begin{aligned}
f(q)= & {\left[x_{1}^{2}+z^{2}-2 x_{1} z \cos \left(60^{\circ}+\alpha\right)\right]^{1 / 2} } \\
& +\left[\left(\sum x-x_{1}\right)^{2}+\left(\sum y-z\right)^{2}-2\left(\sum x-x_{1}\right)\left(\sum y-z\right) \cos \left(60^{\circ}+\alpha\right)\right]^{1 / 2} .
\end{aligned}
$$

Setting

$$
\begin{aligned}
\frac{\partial f(q)}{\partial z}= & {\left[z-x_{1} \cos \left(60^{\circ}+\alpha\right)\right]\left[x_{1}^{2}+z^{2}-2 x_{1} z \cos \left(60^{\circ}+\alpha\right)\right]^{-1 / 2} } \\
& -\left[\left(\sum y-z\right)-\left(\sum x-x_{1}\right) \cos \left(60^{\circ}+\alpha\right)\right] \\
& \cdot\left[\left(\sum x-x_{1}\right)^{2}+\left(\sum y-z\right)^{2}-2\left(\sum x-x_{1}\right)\left(\sum y-z\right) \cos \left(60^{\circ}+\alpha\right)\right]^{-1 / 2}
\end{aligned}
$$

to zero, we obtain

$$
\begin{aligned}
& \left(\frac{z-x_{1} \cos \left(60^{\circ}+\alpha\right)}{\sum y-z-\left(\sum x-x_{1}\right) \cos \left(60^{\circ}+\alpha\right)}\right)^{2} \\
& \quad=\frac{x_{1}^{2}+z^{2}-2 x_{1} z \cos \left(60^{\circ}+\alpha\right)}{\left(\sum x-x_{1}\right)^{2}+\left(\sum y-z\right)^{2}-2\left(\sum x-x_{1}\right)\left(\sum y-z\right) \cos \left(60^{\circ}+\alpha\right)},
\end{aligned}
$$

which can be reduced to

$$
\begin{gathered}
x_{1}^{2}\left[\left(\sum y-z\right)^{2}-2\left(\sum x-x_{1}\right)\left(\sum y-z\right) \cos \left(60^{\circ}+\alpha\right)\right] \\
=\left(\sum x-x_{1}\right)^{2}\left[z^{2}-2 x_{1} z \cos \left(60^{\circ}+\alpha\right)\right] .
\end{gathered}
$$

Clearly, $z=x_{1}\left(\sum y\right)(\Sigma x)^{-1}$ is the unique nonnegative solution.

We now prove the general case by induction on $n+i$. For $n=4, i$ is necessarily 2 and the proof has been given. For $n>4$ and $i>2$, let $\left[p_{1}, q\right]$ meet $\left[p_{i-1}, p_{i}\right]$ at $r$. Suppose that $q$ is not on $\left[p_{1}, p_{n}\right]$; then neither is $r$. Hence

$$
\begin{aligned}
L_{S}\left(P_{n}, \alpha\right)< & L_{S}\left(\left\{p_{1}, p_{2}, \ldots, p_{i-1}, r\right\}, \alpha\right)+L_{S}\left(\left\{r, p_{i}, p_{i+1}, \ldots, p_{n}\right\}, \alpha\right) \\
< & L_{S}\left(\left\{p_{1}, p_{2}, \ldots, p_{i-1}, r\right\}, \alpha\right)+L_{S}\left(\left\{r, p_{i}, q\right\}, \alpha\right) \\
& +L_{S}\left(\left\{q, p_{i+1}, p_{i+2}, \ldots, p_{n}\right\}, \alpha\right) \\
= & L_{S}\left(\left\{p_{1}, p_{2}, \ldots, p_{i}, q\right\}, \alpha\right)+L_{S}\left(\left\{q, p_{i+1} p_{i+2}, \ldots, p_{n}\right\}, \alpha\right),
\end{aligned}
$$

where both inequalities follow from the induction hypothesis. This shows that $q$ is not the minimizing point unless $q$ is on $\left[p_{1}, p_{n}\right]$.

3. The main result. Let $l$ be an edge in an SMT $T$. Then the clockwise path of $l$ is the path starting with $l$ and turning clockwise whenever possible. Similarly, we can define the counterclockwise path of $l$. Notice that both types of paths can end only at regular points. 
REMARKS. For $\alpha \geqslant 120^{\circ}, S_{i}=\left[p_{i}, p_{i+1}\right] \cup\left[p_{i+1}, p_{i+2}\right]$ and $L_{S}\left(P_{n}, \alpha\right)=\Sigma x+\Sigma y$. It is easily verified that $f(q)=L_{S}\left(P_{n}, \alpha\right)$ for all $q$.

THEOREM 3. Suppose that $Z\left(P_{n}, \alpha\right)$ is convex and normal. Then $S$ is its SMT.

Proof. We prove Theorem 3 by induction on $n$. Suppose that $T$ is an SMT for $P_{n}$. Since $\Varangle p_{2} p_{1} p_{3}<180^{\circ}-\Varangle p_{1} p_{2} p_{3}=180^{\circ}-\alpha \leqslant 120^{\circ}$, by Lemmas 1 and $3, p_{1}$ has only one edge. If this edge ends at a regular point $p_{i}$, then

$$
\begin{aligned}
L_{T} & =\left|p_{1}, p_{i}\right|+L_{S}\left(P_{n}-\left\{p_{1}\right\}, \alpha\right) \\
& \geqslant x_{1}+L_{S}\left(P_{n}-\left\{p_{1}\right\}, \alpha\right) \quad \text { by Lemma } 2 \\
& =L_{S}\left(\left\{p_{1}, p_{2}, p_{2}\right\}, \alpha\right)+L_{S}\left(P_{n}-\left\{p_{1}\right\}, \alpha\right) \\
& >L_{S}\left(P_{n}, \alpha\right)
\end{aligned}
$$

by Theorem 2 and by the remarks, hence either $S$ is an SMT or $T$ is not. Therefore we assume that $p_{1}$ is adjacent to a Steiner point $s_{1}$. Furthermore, the counterclockwise path of $\left[p_{1}, s_{1}\right]$ must end at $p_{2}$ for otherwise $p_{2}$ cannot be adjacent to any point. The reason for this is that $p_{2}$ certainly cannot be adjacent to any Steiner point $s_{2}$, for the clockwise path of $\left[p_{2}, s_{2}\right]$ would have nowhere to end. We may assume that $p_{2}$ is adjacent to $p_{3}$, for if $p_{2}$ is adjacent to any other regular point $p_{i}$, then we can replace $\left[p_{2}, p_{i}\right]$ by $\left[p_{2}, p_{3}\right]$ without increasing the length of $T$, by Lemma 2 . But $\left[p_{2}, p_{3}\right]$ will cross the counterclockwise path of $\left[p_{1}, s_{1}\right]$, which violates the minimality of $T$.

Let $H$ denote the counterclockwise path of $\left[p_{1}, s_{1}\right]$ which ends at $p_{2}$ and let $s_{2}$ be the Steiner point adjacent to $p_{2}\left(s_{1}=s_{2}\right.$ is possible) on this path. We now show that we need only consider the case that $H$ has only one Steiner point, i.e., $s_{1}=s_{2}$.

We first prove that $\Varangle s_{2} p_{2} p_{1} \leqslant 120^{\circ}$. Suppose to the contrary that $\Varangle s_{2} p_{2} p_{1}>$ $120^{\circ}$. Case (i). $\left[p_{2}, s_{2}\right]$ meets $\left[p_{3}, p_{4}\right]$ at $w$. Then $\Varangle p_{3} w p_{2}=180^{\circ}-\Varangle w p_{2} p_{1}<$ $60^{\circ}<\alpha$. Therefore we can replace $\left[p_{2}, s_{2}\right]$ by $\left[p_{2}, p_{3}\right]$ and shorten $T$, a contradiction to the minimality of $T$. Case (ii). $\left[p_{2}, s_{2}\right]$ ends before $\left[p_{3}, p_{4}\right]$ (see Figure 3 ).

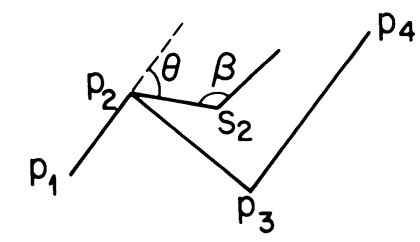

FIGURE 3. $\Varangle s_{2} p_{2} p_{1} \geqslant 120^{\circ}$

It must be the case that $\theta+\beta>180^{\circ}$, for otherwise the counterclockwise path of $\left[p_{2}, s_{2}\right]$ could not reach $\left[p_{3}, p_{4}\right]$ and would have nowhere to end. Furthermore $\beta=120^{\circ}$ by Lemma 2 . Hence $\Varangle s_{2} p_{2} p_{1}=180^{\circ}-\theta<120^{\circ}$.

Next we prove that $\Varangle p_{2} p_{1} s_{1}<60^{\circ}$ (see Figure 4).

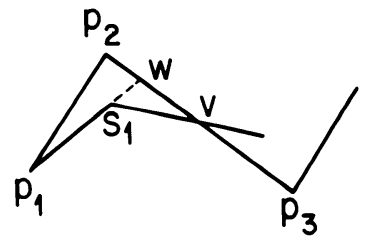

FIGURE 4. $\Varangle p_{2} p_{1} s_{1}<60^{\circ}$ 
Suppose to the contrary that $\Varangle p_{2} p_{1} s_{1} \geqslant 60^{\circ}$. We again consider two cases. Case (i). $\left[p_{1}, s_{1}\right]$ meets $\left[p_{2}, p_{3}\right]$ at $w$. Then

$$
\Varangle p_{1} w p_{2}=180^{\circ}-\alpha-\Varangle p_{2} p_{1} w \leqslant 60^{\circ} \leqslant \alpha .
$$

Therefore $\left|p_{1}, w\right| \geqslant\left|p_{1}, p_{2}\right|$ and we can replace $\left|p_{1}, w\right|$ by $\left[p_{1}, p_{2}\right]$ and obtain a Steiner tree not longer than $T$. But we have already shown that $p_{1}$ cannot be adjacent to $p_{2}$ in an SMT, hence $T$ is not minimal. Case(ii). $\left[p_{1}, s_{1}\right]$ ends before $\left[p_{2}, p_{3}\right]$. Let $\left[s_{1}, v\right]$ denote the edge at $s_{1}$ not on $H$. Then $\left[s_{1}, v\right]$ must meet $\left[p_{2}, p_{3}\right]$ or else the clockwise path of $\left[p_{1}, s_{1}\right]$ would have nowhere to end. Let the extension of $\left[p_{1}, s_{1}\right]$ meet $\left[p_{2}, p_{3}\right]$ at $w$. Then

$$
120^{\circ}=\Varangle p_{1} s_{1} v>\Varangle p_{1} w p_{3}=\alpha+\Varangle p_{2} p_{1} s_{1},
$$

hence $\Varangle p_{2} p_{1} s_{1}<60^{\circ}$.

We have proven that $\Varangle s_{2} p_{2} p_{1}+\Varangle p_{2} p_{1} s_{1}<120^{\circ}+60^{\circ}=180^{\circ}$. Suppose that there are $k$ Steiner points on $H$. Then since all angles of $H$ are $120^{\circ}$,

$$
\Varangle s_{2} p_{2} p_{1}+\Varangle p_{2} p_{1} s_{1}=k \cdot\left(180^{\circ}-120^{\circ}\right)=k \cdot 60^{\circ} \text {. }
$$

Hence $k$ must be either one or two.

We first study the special case $n=4$.

Claim. For $n=4, k=1$ in $T$, with the single exception that, if $\alpha=60^{\circ}$ and $x_{1}=x_{2}=y_{1}$, then there are two SMT's with $k=1$ in one and $k=2$ in the other.

Proof. Let $\left[p_{1}, p_{4}\right]$ meet $\left[p_{2}, p_{3}\right]$ at $q$ (see Figure 5).

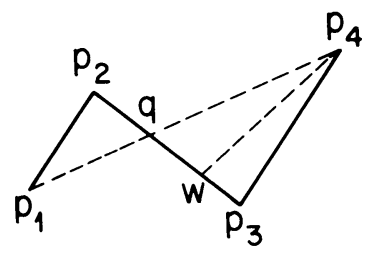

Figure 5. A $Z\left(P_{4}, \alpha\right)$

Without loss of generality assume $\left|p_{2}, q\right| \leqslant\left|p_{3}, q\right|$. Let $w$ be a point on $\left[p_{3}, q\right]$ such that $\left|p_{2}, q\right|=|w, q|$. Since $\Varangle p_{4} w p_{2} \geqslant \alpha \geqslant \Varangle p_{4} p_{2} w$ (the second inequality is due to the "normal" assumption), $\left|w, p_{4}\right| \leqslant\left|p_{2}, p_{4}\right|$. It follows easily from the cosine law that $\Varangle p_{4} q w \leqslant \Varangle p_{4} q p_{2}$. Furthermore, equality can take place only when $\alpha=60^{\circ}$ and $x_{1}=x_{2}=y_{1}$. By noting that the union of the SMT for $\left\{p_{1}, p_{2}, q\right\}$ and the SMT for $\left\{q, p_{3}, p_{4}\right\}$ is a full Steiner tree for $\left\{p_{1}, p_{2}, p_{3}, p_{4}\right\}$ with $k=1$, the Claim follows immediately from Lemma 8.

Next we show that for $n \geqslant 5, k=1$ in $T$. Suppose to the contrary that $k=2$ in $T$ (see Figure 6).

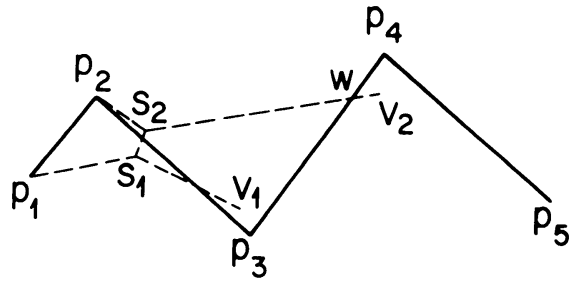

FIGURE 6. $k=2$ and $n \geqslant 5$ 
We first prove that $\left[p_{1}, s_{1}\right]$ cannot meet $\left[p_{3}, p_{4}\right]$. Suppose to the contrary that they meet at $c$. Then $\Varangle p_{1} c p_{3}=\Varangle p_{2} p_{1} c<60^{\circ} \leqslant \alpha<\Varangle c p_{3} p_{1}$. Therefore $\left|p_{1}, c\right|>$ $\left|p_{1}, p_{3}\right|>\left|p_{1}, p_{2}\right|$, a contradiction to the minimality of $T$ by Lemma 5 .

Another fact we wish to point out is that the third edge at $s_{2}$, say $\left[s_{2}, v_{2}\right]$, must meet $\left[p_{3}, p_{4}\right]$. If not, let the extension of $\left[s_{2}, v_{2}\right]$ meet $\left[p_{3}, p_{4}\right]$ at $w$. Then

$$
\Varangle s_{2} w p_{4}=180^{\circ}-\Varangle s_{2} w p_{3}=180^{\circ}-\Varangle p_{2} p_{1} s_{1}>120^{\circ} .
$$

Therefore the counterclockwise path of $\left[s_{2}, v_{2}\right]$ cannot reach $p_{4}$ and can end nowhere.

Let $\left[s_{1}, v_{1}\right]$ denote the third edge at $s_{1}$. Let $\left[s_{2}, v_{2}\right]$ meet $\left[p_{3}, p_{4}\right]$ at $w$. Suppose that $\left[s_{1}, v_{1}\right]$ meets $\left[p_{3}, p_{4}\right]$ at $z$. Let $\left[p_{1}, w\right]$ meet $\left[p_{2}, z\right]$ at $q^{\prime}$. Then it is easily verified that $\Varangle p_{1} q^{\prime} p_{2} \leqslant \Varangle p_{1} q p_{2}$. By Lemma 8 , the part of $T$ connecting $p_{1}, p_{2}$, $z, w$ can be shortened, a contradiction to the minimality of $T$, except possibly when $\alpha=60^{\circ}, x_{1}=y_{1}\left(=x_{2}\right), z=p_{3}$, and $w=p_{4}$. Suppose that $p_{3}$ has a second edge in $T$. This edge must meet $\left[p_{4}, p_{5}\right]$, say at $x$, or else the counterclockwise path of this edge would have nowhere to end. But $\Varangle x p_{3} p_{4} \leqslant \Varangle p_{5} p_{3} p_{4} \leqslant \alpha$ implies that $\left|p_{4}, x\right| \leqslant\left|p_{3}, x\right|$, hence we can replace $\left[p_{3}, x\right]$ by $\left[p_{4}, x\right]$ without increasing the length of $T$. It follows that we can assume $T$ is a combination of the tree $S_{3} \cup S_{4} \cup \cdots \cup S_{n-2}$ on $P^{\prime \prime}=\left\{p_{4}, p_{5}, \ldots, p_{n}\right\}$ and an SMT on $P^{\prime}=$ $\left\{p_{1}, p_{2}, p_{3}, p_{4}\right\}$. Replacing the subtree of $T$ connecting $P^{\prime}$ by the other full Steiner tree, i.e., $S_{1} \cup S_{2}$, preserves the length of the tree according to the Claim. On the other hand Theorem 2 says that $L_{S}\left(P^{\prime}, 60^{\circ}\right)+L_{S}\left(P^{\prime \prime}, 60^{\circ}\right)>L_{S}\left(P, 60^{\circ}\right)$. Hence $T$ is not minimal.

Next consider the case that $\left[s_{1}, v_{1}\right]$ does not meet $\left[p_{3}, p_{4}\right]$. Let $\left[v_{1}, u\right]$ denote the edge at $v_{1}$ parallel to $\left[s_{2}, v_{2}\right]$. Note that the counterclockwise path of $\left[v_{1}, u\right]$ cannot end at $p_{4}$, since $\left[s_{2}, v_{2}\right]$ or its counterclockwise path ends there. Therefore $\left[v_{1}, u\right]$ must meet $\left[p_{4}, p_{5}\right]$ or else its counterclockwise path would have nowhere to end. Suppose that $\left[v_{1}, u\right]$ meets $\left[p_{3}, p_{4}\right]$ and $\left[p_{4}, p_{5}\right]$ at $t$ and $z$, respectively. Since $\Varangle p_{4} t z=\Varangle p_{2} p_{1} s_{1}<60^{\circ} \leqslant \alpha$, it follows that $\left|v_{1} u\right|>|t, z|>\left|p_{4}, z\right|$. Therefore we can delete $\left[v_{1}, u\right]$ and add $\left[p_{4}, z\right]$ to shorten $T$, a contradiction to the minimality of $T$.

We have shown that $k \neq 2$. Therefore $k=1$, i.e., $s_{1}$ and $s_{2}$ are the same point $s$. Note that $s$ must lie within $\triangle p_{1} p_{2} p_{3}$, for if $s$ lies above $\left[p_{2}, p_{3}\right]$, then $\Varangle s p_{2} p_{1}+$ $p_{2} p_{1} s>\alpha \geqslant 60^{\circ}$, a contradiction to the fact that $k=1$. Let $[s, v]$ denote the third edge at $s$. Then $[s, v]$ must meet $\left[p_{2}, p_{3}\right]$ for otherwise the clockwise path of $[s, v]$ cannot reach $p_{3}$ and has no place to end. Next we prove that $p_{2}$ cannot have a second edge. Suppose to the contrary that $\left[p_{2}, w\right], w \neq s$, exists. Then $\left[p_{2}, w\right]$ must meet $\left[p_{3}, p_{4}\right]$ at $z$, for otherwise the counterclockwise path of $\left[p_{2}, w\right]$ clearly would have nowhere to end (noting that $\Varangle p_{1} p_{2} w>\Varangle s_{1} p_{2} w \geqslant 120^{\circ}$ ). Now $\left|p_{2}, p_{4}\right| \geqslant$ $\left|p_{3}, p_{4}\right|$ implies $\alpha \geqslant \Varangle p_{4} p_{2} p_{3}>\Varangle z p_{2} p_{3}$, hence $\left|p_{2}, z\right|>\left|p_{3}, z\right|$. Therefore we can replace $\left[p_{2}, z\right]$ by $\left[p_{3}, z\right]$ and shorten $T$, a contradiction to the minimality of $T$. We conclude that $p_{2}$ has only one edge.

Let $[s, v]$ meet $\left[p_{2}, p_{3}\right]$ at $q$. Then $T$ is partitioned into a subtree connecting the three points $p_{1}, p_{2}, q$ and a subtree connecting $q, p_{3}, \ldots, p_{n}$. By Theorem $2, q$ must lie on $\left[p_{1}, p_{n}\right]$ and the induction hypothesis brings the conclusion $T=S$. 
COROLlaRY. The length of a Steiner minimal tree on $Z\left(P_{n}, \alpha\right)$ is

$$
\left\{\begin{array}{l}
{\left[\left(\sum x\right)^{2}+\left(\sum y\right)^{2}-2\left(\sum x\right)\left(\sum y\right) \cos \left(60^{\circ}+\alpha\right)\right]^{1 / 2} \text { if } 60^{\circ} \leqslant \alpha \leqslant 120^{\circ},} \\
\sum x+\sum y \text { if } 120^{\circ} \leqslant \alpha \leqslant 180^{\circ} .
\end{array}\right.
$$

4. Some concluding remarks. Smith [7] studied $Z\left(P_{n}, \alpha\right)$ for the case $\alpha=60^{\circ}$ and all $x_{i}$ and $y_{j}$ are equal, say, of length one. He obtained SMT's for $n \leqslant 9$ and conjectured that for $n=2 k+1$, the length of a Steiner minimal tree is $\sqrt{3} k$. Applying the formula given in the Corollary of Theorem 3 to this special case, we obtain the length of an SMT to be $\sqrt{3} k$ if $n=2 k+1$, and $\left[3\left(k^{2}+k\right)+1\right]^{1 / 2}$ if $n=2 k+2$. Therefore, Smith's conjecture is proved.

\section{REFERENCES}

1. W. M. Boyce, An improved program for the full Steiner tree problem, Bell Lab. Tech. Memo., 1975.

2. F. R. K. Chung and R. L. Graham, Steiner trees for ladders, Ann. Discrete Math. 2 (1978), 173-200.

3. M. R. Garey, R. L. Graham and D. S. Johnson, The complexity of computing Steiner minimal trees, SIAM J. Appl. Math. 32 (1977), 835-859.

4. E. N. Gilbert and H. O. Pollak, Steiner minimal trees, SIAM J. Appl. Math. 16 (1968), 1-29.

5. Z. A. Melzak, On the problem of Steiner, Canad. Math. Bull. 4 (1960), 143-148.

6. H. O. Pollak, Some remarks on the Steiner problem, J. Combin. Theory Ser. A 24 (1978), 278-295.

7. J. M. Smith, Point set decomposition and suboptimal Steiner minimal trees, paper presented at the TIMS-ORSA Meeting, Colorado Springs, Colo., 1980.

Institute of Applied Mathematics, Academia Sinica, Beijing, China

Bell laboratories, Murray Hill, New Jersey 07078 (Current address of F. K. Hwang)

Baoshan Iron and Steel General Works, Shanghai, China

Current address (D. Z. Du): Department of Mathematics, University of California at Santa Barbara, Santa Barbara, California 93106

Current address (J. F. Weng): Käfertaler Str. 258, (BBC) Abt. IA/MW4, 6800 Mannheim 1, B.R.D. (West Germany) 